\title{
Cytotoxicity and immunological responses following oral vaccination of nanoencapsulated avian influenza virus H5 DNA vaccine with green synthesis silver nanoparticles.
}

\begin{abstract}
DNA formulations provide the basis for safe and cost effective vaccine. Low efficiency is often observed in the delivery of DNA vaccines. In order to assess a new strategy for oral DNA vaccine formulation and delivery, plasmid encoding hemagglutinin (HA) gene of avian influenza virus, A/Ck/Malaysia/5858/04 (H5N1) (pcDNA3.1/H5) was formulated using green synthesis of sliver nanoparticles (AgNP) with polyethylene glycol (PEG). AgNP were successfully synthesized uniformly dispersed with size in the range of 4 to $18 \mathrm{~nm}$ with an average size of $11 \mathrm{~nm}$. Cytotoxicity of the prepared AgNP was investigated in vitro and in vivo using MCF-7 cells and cytokine expression, respectively. At the concentration of -5 $\log 10 \mathrm{AgNP}$, no cytotoxic effects were detected in MCF-7 cells with $9.5 \%$ cell death compared to the control. One-day-old specific pathogen-free (SPF) chicks immunized once by oral gavage with $10 \mu \mathrm{l}$ of pcDNA3.1/H5 (200 ng/ml) nanoencapsulated with $40 \mu \mathrm{l} \mathrm{AgNP}$ $(3.7 \times 10-2 \mu \mathrm{g}$ of $\mathrm{Ag})$ showed no clinical manifestations. PCR successfully detect the AgNP/H5 plasmid from the duodenum of the inoculated chicken as early as $1 \mathrm{~h}$ postimmunization. Immunization of chickens with $\mathrm{AgNP} / \mathrm{H} 5$ enhanced both pro inflammatory and Th1-like expressions, although no significant differences were recorded in the chickens inoculated with $\mathrm{AgNP}, \mathrm{AgNP} / \mathrm{pcDNA} 3.1$ and the control. In addition, serum samples collected from immunized chickens with AgNP/H5 showed rapidly increasing antibody against $\mathrm{H} 5$ on day 14 after immunization. The highest average antibody titres were detected on day 35 post-immunization at $51.2 \pm 7.5$. AgNP/H5 also elicited both CD4+ and CD8+ T cells in the immunized chickens as early as day 14 after immunization, at $7.5 \pm 2.0$ and $20 \pm$ 1.9 percentage, respectively. Hence, single oral administrations of AgNP/H5 led to induce both the antibody and cell-mediated immune responses as well as enhanced cytokine production.
\end{abstract}

Keyword: Avian influenza virus; H5 DNA vaccine; HI antibody; T cell; Cytokine; Green synthesis AgNP 